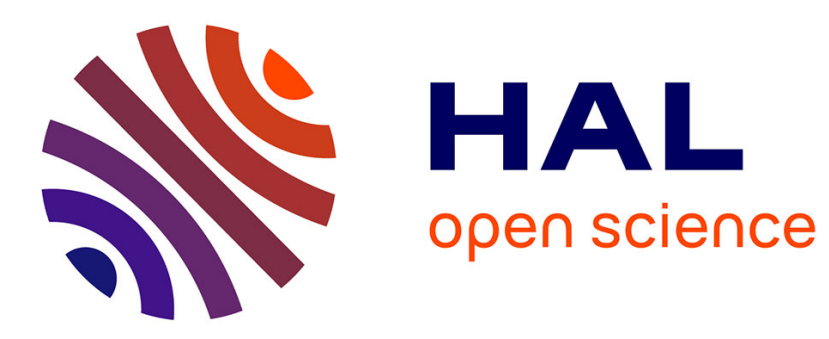

\title{
The two-dimensional one-component plasma in an inhomogeneous background: exact results
}

\author{
A. Alastuey, J.L. Lebowitz
}

\section{To cite this version:}

A. Alastuey, J.L. Lebowitz. The two-dimensional one-component plasma in an inhomogeneous background: exact results. Journal de Physique, 1984, 45 (12), pp.1859-1874. 10.1051/jphys:0198400450120185900 . jpa-00209930

\section{HAL Id: jpa-00209930 https://hal.science/jpa-00209930}

Submitted on 1 Jan 1984

HAL is a multi-disciplinary open access archive for the deposit and dissemination of scientific research documents, whether they are published or not. The documents may come from teaching and research institutions in France or abroad, or from public or private research centers.
L'archive ouverte pluridisciplinaire HAL, est destinée au dépôt et à la diffusion de documents scientifiques de niveau recherche, publiés ou non, émanant des établissements d'enseignement et de recherche français ou étrangers, des laboratoires publics ou privés. 
Classification

Physics Abstracts

$05.20-50.00$

\title{
The two-dimensional one-component plasma in an inhomogeneous background : exact results
}

\author{
A. Alastuey \\ Laboratoire de Physique Théorique et Hautes Energies (*), Université de Paris-Sud, \\ 91405 Orsay Cedex, France \\ and J. L. Lebowitz \\ Department of Mathematics, Rutgers University, New Brunswick, New Jersey 08903, U.S.A.
}

(Reçu le 9 juillet 1984, accepté le 22 août 1984)

\begin{abstract}
Résumé. - Nous étudions le jellium inhomogène en deux dimensions où la densité du fond varie arbitrairement dans une seule direction. A $\Gamma=2$, les densités à un et deux corps des particules sont représentées par des fonctionnelles explicites du potentiel électrostatique créé par le fond. Le présent modèle peut être utilisé pour décrire une large variété de surfaces chargées.
\end{abstract}

\begin{abstract}
We study the general inhomogeneous two-dimensional jellium where the background density varies in one space direction only. At $\Gamma=2$, explicit functional representations of the one- and two-body densities of the particles are derived in terms of the electrostatic potential created by the background. The present model can be used for describing a large variety of charged interfaces.
\end{abstract}

\section{Introduction.}

There has been and continues to be much interest in the properties of inhomogeneous charged systems. The motivations for this are as many as the physical situations in which the non-uniformities play a rôle from Astrophysics to electrical double layer in electrochemistry and biology. In addition, it is sometimes convenient and justified, even in overall uniform plasmas, to consider the fast moving light electrons as being in equilibrium in the non-uniform potential produced by a specified fixed configuration of the heavier ions. This occurs for example in the study of line shapes of radiators in a plasma.

The simplest model describing a charged system is based on the one-component plasma (jellium) : identical particles with charge $e$ move in a rigid background with opposite charge. Recently the microscopic structures of various « surfaces » in this model have been worked out exactly in two dimensions at the special temperature $T$, for which the coupling constant $\Gamma=e^{2} / k_{\mathrm{B}} T=2[1-6]$. These exact calculations involve

(*) Laboratoire Associé au Centre National de la Recherche Scientifique. random-matrix methods [7-8] similar to those used for studying the bulk properties of the two-dimensional jellium at $\Gamma=2[9-10]$.

In this paper, we extend the previous exact calculations to the case where the background density has an arbitrary shape in one direction $\rho_{\mathrm{B}}(x, y)=\rho_{\mathrm{B}}(x)$. In addition there can also be an external potential $V_{\text {ext }}(x)$ applied to the particles. At $\Gamma=2$, we derive explicit functional representations of the one- and two-body densities of the particle in terms of $V_{\text {ext }}(x)$ and of the electrostatic potential created by the background.

We proceed as follows : in $\S 2$, we consider the case where $\rho_{\mathrm{B}}(x) \rightarrow \rho_{1}\left(\rho_{2}\right)$ as $x \rightarrow-\infty(\infty)$. We use a method very similar to the one introduced by Jancovici [4] for the step case, $\rho_{\mathrm{B}}(x)=\rho_{1}$ for $x<0$ and $\rho_{\mathrm{B}}(x)=\rho_{2}$ for $x>0$. The density profile of the particles, as well as the truncated two-body distribution function, are computed in terms of simple integral representations. In $\S 3$, we specialize our expressions to the case $\rho_{1}=\rho_{2}$. The corresponding integral representations are shown to remain valid when $\rho_{\mathrm{B}}(x)$ is a periodic function, in $\S 4$. Finally, in $\S 5$, we briefly indicate how to extend the expressions derived in $\S 2,3$ and 4 to the cases where an external potential is present. 
Throughout the paper, we systematically discuss general sum rules for inhomogeneous charged systems [11-14]. A few examples corresponding to simple forms of $\rho_{\mathrm{B}}(x)$ and $V_{\text {ext }}(x)$ are studied in detail.

\section{The smooth step.}

2.1 Formulation OF THE PROBlem. - We start from a finite system with circular symmetry : $N$ particles with charge $e$ in a disk with radius $R$ and volume $\Lambda$. The disk is filled with a background charge density $-e \rho_{\mathrm{B}}(r)$ such that

$$
\text { and } \left.\begin{array}{lll}
\rho_{\mathrm{B}}(r)=\rho & \text { for } \quad r \leqslant R_{\min } \\
& \rho_{\mathrm{B}}(r)=\rho(1-\alpha) & \text { for } \quad R_{\max } \leqslant r \leqslant R,
\end{array}\right\}
$$

where $0<\alpha<1$ and $\rho_{\mathrm{B}}(r)$ is continuous for $r<R$. Two particles $i$ and $j$ interact through the two-dimensional Coulomb potential

$$
v\left(r_{i j}\right)=-e^{2} \ln \left(r_{i j} / L_{\mathrm{s}}\right)
$$

( $L_{\mathrm{s}}$ is an irrelevant scale length). Let $V_{\mathrm{B}}\left(r_{i}\right)$ be the interaction potential of particle $i$ with the background minus the corresponding quantity at $r_{i}=0$, and let $V$ be the total interaction potential of the system. For the inverse temperature $\beta$ such that $\Gamma=\beta e^{2}=2$, the Boltzmann factor can be expressed in terms of a Vandermonde determinant [9-10], namely

$$
\begin{array}{r}
\exp \left[-\beta V\left(\mathbf{r}_{1}, \ldots, \mathbf{r}_{N}\right)\right]=A \exp \left[-\beta \sum_{i=1}^{N} V_{\mathbf{B}}\left(r_{i}\right)\right] \\
\times|\operatorname{Det}| \begin{array}{lll}
z_{1}^{0} & \ldots & z_{N}^{0} \\
\vdots & & \vdots \\
z_{1}^{N-1} & \ldots & z_{N}^{N-1}
\end{array} \mid \|^{2}
\end{array}
$$

( $A$ is a constant), where $z_{j}$ is the complex number $r_{j} \exp \left(i \theta_{j}\right)$ and $\left(r_{j}, \theta_{j}\right)$ are the polar coordinates of $\mathbf{r}_{j}$ in a given frame.

Since $V_{B}$ has cylindrical symmetry, the angular integration of the Boltzmann factor (2.3) upon any set $\left\{\theta_{j}\right\}$ can be performed explicitly. Expanding the Vandermonde determinant and using the orthogonality of the functions $\exp \left(i n \theta_{j}\right)$, the $n$-body distribution function of the particles becomes [7-8]

$$
\begin{aligned}
& \rho^{(n)}\left(\mathbf{r}_{1}, \ldots, \mathbf{r}_{n}\right)=\exp \left[-\beta \sum_{i=1}^{n} V_{\mathrm{B}}\left(r_{i}\right)\right] \times \\
& \text { Det }\left|K_{N, \Lambda}\left(z_{i} z_{j}^{*}\right)\right|,
\end{aligned}
$$

where $K_{N . A}(Z)$ is defined by

$$
K_{N, \Lambda}(Z)=\sum_{p=0}^{N-1} \frac{Z^{p}}{\int_{\Lambda} \mathrm{dr} r^{2 p} \exp \left[-\beta V_{\mathrm{B}}(r)\right]}
$$

For studying the microscopic structure of the plane interface, we have to take in (2.4) the successive limits, $R \rightarrow \infty, N \rightarrow \infty$, and finally $R_{\min }$ and $R_{\max } \rightarrow \infty$, the differences $\left(R_{\max }-R_{\min }\right)$ and $\left(r_{j}-R_{\min }\right)$ being kept fixed. The first two limits are somewhat trivial. The last one requires a suitable adaptation of the technique first introduced by Jancovici [4].

2.2 THE ONE-BODY DENSITY. - In the following all the distances are redefined in units of $a=1 /(\pi \rho)^{1 / 2}$ and the $n$-body distribution functions are redefined in units of $\rho^{n}$. Once the limits $R \rightarrow \infty$ and $N \rightarrow \infty$ have been taken, the one-body density is given by

$$
\rho\left(r_{1}\right)=\exp \left[-\beta V_{\mathbf{B}}\left(r_{1}\right)\right] K\left(r_{1}^{2}\right),
$$

where the function $K$ of the dimensionless complex variable $Z$ is related to $(2.5)$ by

$$
K(Z)=\frac{1}{\rho} \lim _{N \rightarrow \infty} \lim _{R \rightarrow \infty} K_{N, \Lambda}\left(Z a^{2}\right)=\sum_{p=0}^{\infty} \frac{Z^{p}}{D(p)},
$$

with

$$
D(p)=2 \int_{0}^{\infty} \mathrm{d} r r^{2 p+1} \exp \left[-\beta V_{\mathrm{B}}(r)\right]
$$

It is clear that for a given continuous density $\lambda(r)$, $0 \leqslant r \leqslant R_{\max }-R_{\min }$, such that $\rho_{\mathrm{B}}\left(R_{\min }+r\right)=\lambda(r)$, the average density $\left(\pi R^{2}\right)^{-1} \int_{r \leqslant R} \mathrm{dr} \rho_{\mathrm{B}}(r)$ will, for $R_{\min }$ and $R$ sufficiently large and $\left(R_{\max }-R_{\min }\right)$ fixed, always lie between $\rho_{1}$ and $\rho_{2}$. We can then find some $R_{0}<R$ such that the continuous density $\rho_{\mathrm{B}}(r)$ is given by

$\rho_{\mathrm{B}}(r)=q_{\mathrm{B}}(r)+\rho H\left(R_{0}-r\right)+\rho(1-\alpha) H\left(r-R_{0}\right)$,

where $H(x)$ is the Heaviside function $H(x)=0$ for $x<0$ and $H(x)=1$ for $x>0$ and $\int_{r \leqslant R} \mathrm{dr} q_{\mathrm{B}}(r)=0$. Then, $\beta V_{\mathrm{B}}(r)$ becomes

$$
\left.\begin{array}{l}
\beta V_{\mathrm{B}}(r)=r^{2}+\phi(r) \quad \text { for } \quad r \leqslant R_{0}, \\
\beta V_{\mathrm{B}}(r)=R_{0}^{2}+2 \alpha R_{0}^{2} \ln \left(r / R_{0}\right)+(1-\alpha)\left(r^{2}-R_{0}^{2}\right)+\phi(r) \quad \text { for } \quad R_{0} \leqslant r,
\end{array}\right\}
$$


with

$$
\phi(r)=4 \int_{0}^{r} \mathrm{~d} r^{\prime} \frac{\int_{0}^{r^{\prime}} \mathrm{d} r^{\prime \prime} r^{\prime \prime} q_{\mathrm{B}}\left(r^{\prime \prime}\right)}{r^{\prime}}
$$

$\left(q_{\mathrm{B}}(r)\right.$ is redefined in units of $\left.\rho\right) . q_{\mathrm{B}}(r)$ has a compact support, i.e. $q_{\mathrm{B}}(r)=0$ for $r \leqslant R_{\min }$ or $R_{\max } \leqslant r\left({ }^{1}\right)$. Therefore, we have

$$
\begin{aligned}
& \phi(r)=0 \quad \text { for } \quad r \leqslant R_{\min }, \\
& \phi(r)=4 \int_{R_{\min }}^{r} \mathrm{~d} r^{\prime} \frac{\int_{R_{\min }}^{r^{\prime}} \mathrm{d} r^{\prime \prime} r^{\prime \prime} q_{\mathrm{B}}\left(r^{\prime \prime}\right)}{r^{\prime}} \quad \text { for } \quad R_{\min } \leqslant r \leqslant R_{\max }, \\
& \phi(r)=\text { const. }=4 \int_{R_{\min }}^{R_{\max }} \mathrm{d} r^{\prime} \frac{\int_{R_{\min }}^{r^{\prime}} \mathrm{d} r^{\prime \prime} r^{\prime \prime} q_{\mathrm{B}}\left(r^{\prime \prime}\right)}{r^{\prime}} \quad \text { for } \quad R_{\max } \leqslant r .
\end{aligned}
$$

We take now the final limit $R_{\min } \rightarrow \infty$ defined in $\S 2.1$. In this limit, the differences $\left(R_{0}-R_{\min }\right)$, $\left(R_{\max }-R_{\min }\right)$ remain finite. Furthermore, putting $x=r-R_{0}$, we have

$$
\int_{d_{<}}^{d_{>}} \mathrm{d} x q_{\mathrm{B}}(x)=0,
$$

where $d_{<}=\lim \left(R_{\min }-R_{0}\right)$ and $d_{>}=\lim \left(R_{\max }-R_{0}\right)$. Neglecting terms of order $1 / R_{0},(2.12)$ becomes

$$
\left.\begin{array}{lll}
\phi(x)=0 & \text { for } & x \leqslant d_{<}, \\
\phi(x)=4 \int_{d_{<}}^{x} \mathrm{~d} x^{\prime} \int_{d_{<}}^{x^{\prime}} \mathrm{d} x^{\prime \prime} q_{\mathrm{B}}\left(x^{\prime \prime}\right) & \text { for } & d_{<} \leqslant x \leqslant d_{>}, \\
\text {const. }=4 \int_{d_{<}}^{d_{>}} \mathrm{d} x^{\prime} \int_{d_{<}}^{x^{\prime}} \mathrm{d} x^{\prime \prime} q_{\mathrm{B}}\left(x^{\prime \prime}\right) & \text { for } & d_{>} \leqslant x .
\end{array}\right\}
$$

The essential point of the present method is that the limit (2.14) of $\phi(r)$ is a continuous bounded function. Then, the remaining part of the calculation is a straightforward extension of a previous work [4]. Since $x_{1}=r_{1}-R_{0}$ remains finite, the dominant contributions to $K\left(r_{1}^{2}\right)$ come from $p=R_{0}^{2}+s R_{0} \sqrt{2}$. For evaluating the corresponding $D(p)$, we first rewrite (2.8) as

$$
\begin{aligned}
D(p)=\int_{0}^{R_{0}^{2}} \mathrm{~d} t \exp [-t+p \ln t] \exp [-\phi(\sqrt{t})]+\exp \left[-\alpha R_{0}^{2}\left(1-\ln R_{0}^{2}\right)\right] \times \\
\times \int_{R_{0}^{2}}^{\infty} \mathrm{d} t \exp \left[-(1-\alpha) t-\alpha R_{0}^{2} \ln t+p \ln t\right] \exp [-\phi(\sqrt{t})] .
\end{aligned}
$$

Since $\exp [-\phi(\sqrt{t})]$ is a slowly-varying function which remains bounded when $R_{0} \rightarrow \infty$, both integrals of (2.15) can be computed by steepest descent methods. Expanding $[-t+p \ln t]$ and $\left[-(1-\alpha) t-\alpha R_{0}^{2} \ln t+p \ln t\right]$ around their respective maxima $t=p$ and $t=\left(p-\alpha R_{0}^{2}\right) /(1-\alpha)$ gives

$$
\begin{aligned}
D(p) \sim R_{0} & \sqrt{2} \exp \left(R_{0}^{2} \ln R_{0}^{2}-R_{0}^{2}+R_{0} s \sqrt{2} \ln R_{0}^{2}\right) \times \\
& \times\left\{\exp \left(s^{2}\right) \int_{-\infty}^{-s} \mathrm{~d} u \exp \left[-u^{2}-\phi\left(\frac{s+u}{\sqrt{2}}\right)\right]\right. \\
& \left.+\frac{1}{\sqrt{(1-\alpha)}} \exp \left[\frac{s^{2}}{(1-\alpha)}\right] \int_{-s / V(1-\alpha)}^{\infty} \mathrm{d} u \exp \left[-u^{2}-\phi\left(\frac{s \sqrt{2}+u \sqrt{2(1-\alpha)}}{2(1-\alpha)}\right)\right]\right\}
\end{aligned}
$$

( $\left.{ }^{1}\right)$ For some particular shapes of $\rho_{\mathrm{B}}(r), R_{0}$ does not belong to $\left[R_{\min }, R_{\max }\right]$. The support of $q_{\mathrm{B}}(r)$ is not $\left[R_{\min }, R_{\max }\right]$ in these cases. This circumstance does not modify the remaining part of the present derivation. 
Noting that

$$
r_{1}^{2 p} \exp \left[-\beta V_{\mathrm{B}}\left(r_{1}\right)\right] \sim \exp \left[R_{0}^{2} \ln R_{0}^{2}-R_{0}^{2}+s R_{0} \sqrt{2} \ln R_{0}^{2}-2 x_{1}^{2}+2 x_{1} s \sqrt{2}-\phi\left(x_{1}\right)\right]
$$

for $x_{1}<0$ and

$$
r_{1}^{2 p} \exp \left[-\beta V_{\mathrm{B}}\left(r_{1}\right)\right] \sim \exp \left[R_{0}^{2} \ln R_{0}^{2}-R_{0}^{2}+s R_{0} \sqrt{2} \ln R_{0}^{2}-2(1-\alpha) x_{1}^{2}+2 x_{1} s \sqrt{2}-\phi\left(x_{1}\right)\right]
$$

for $x_{1}>0$, and replacing the sum over $p$ in $K\left(r_{1}^{2}\right)$ by an integral upon $s$, we finally obtain

$$
\rho(x)=g(x) \exp [-\phi(x)] \int_{-\infty}^{\infty} \mathrm{d} s \exp (2 x s \sqrt{2})\left\{\exp \left(s^{2}\right) \psi_{<}(s)+\exp \left[\frac{s^{2}}{(1-\alpha)}\right] \psi_{>}(s)\right\}^{-1},
$$

with

$$
\begin{array}{ll}
g(x)=\exp \left(-2 x^{2}\right) & \text { for } x \leqslant 0 \\
g(x)=\exp \left[-2(1-\alpha) x^{2}\right] & \text { for } 0 \leqslant x,
\end{array}
$$

and

$$
\begin{aligned}
& \psi_{<}(s)=\int_{-\infty}^{-s} \mathrm{~d} u \exp \left[-u^{2}-\phi\left(\frac{s+u}{\sqrt{2}}\right)\right] \\
& \psi_{>}(s)=\frac{1}{\sqrt{(1-\alpha)}} \int_{-s / \sqrt{11-\alpha)}}^{\infty} \mathrm{d} u \exp \left[-u^{2}-\phi\left(\frac{s+u \sqrt{(1-\alpha)}}{(1-\alpha) \sqrt{2}}\right)\right] .
\end{aligned}
$$

(2.19) expresses $\rho(x)$ as an explicit functional of $\phi(x)$, which is the electrostatic potential in $k_{\mathrm{B}} T$ units (apart from an irrelevant constant) created by the layered charge distribution with charge density $-e q_{\mathrm{B}}(x)$.

Equation (2.19) was derived for a background density varying in the finite interval $\left[d_{<}, d_{>}\right]$but it can be obviously extended to the case $d_{<}=-\infty$ and $d_{>}=\infty$ provided that $q_{\mathrm{B}}(x)$ and $x q_{\mathrm{B}}(x)$ are integrable at infinity and that

$$
\int_{-\infty}^{\infty} \mathrm{d} x q_{\mathrm{B}}(x)=0
$$

When $q_{\mathrm{B}}(x)$ vanishes identically, we recover Jancovici's result [4].

It can be easily checked that $\rho(x)$ goes to 1 or to $(1-\alpha)$ when $x \rightarrow-\infty$ or $\infty$ respectively. The asymptotic forms of $\rho(x)-\rho( \pm \infty)$ depend on the way in which $q_{\mathrm{B}}(x)$ goes to zero at infinity. If $q_{\mathrm{B}}(x)$ has a compact support, $\rho(x)-\rho( \pm \infty)$ is essentially Gaussian. If $q_{\mathrm{B}}(x)$ decays exponentially when $|x| \rightarrow \infty$, so does $\rho(x)-\rho( \pm \infty)$. Finally, if $q_{\mathrm{B}}(x)$ decays algebraically, $\rho(x)-1$ and $\rho(x)-1+\alpha$ behave likewise when $|x| \rightarrow \infty$. In Appendix 1, we show that $\rho(x)$ satisfies the overall neutrality condition

$$
\int_{-\infty}^{\infty} \mathrm{d} x\left[\rho(x)-\rho_{\mathbf{B}}(x)\right]=0
$$

as well as the electrochemical potential balance [11] which reads here

$$
\int_{-\infty}^{\infty} \mathrm{d} x x\left[\rho(x)-\rho_{\mathrm{B}}(x)\right]=-\frac{1}{8} \ln (1-\alpha) .
$$

The integral representation of $\rho(x)$ can be extended to the case $\alpha=1$ by taking the limit $\alpha \rightarrow 1^{-}$in (2.19). First, we break up the integral involved in (2.19) into $\int_{-\infty}^{0} \mathrm{~d} s \ldots+\int_{0}^{\infty} \mathrm{d} s \ldots . . \mathrm{In} \int_{0}^{\infty} \mathrm{d} s \ldots$, we make the variable change $s=t \sqrt{(1-\alpha)}$. Since $\phi$ is bounded on $[-\infty$, $\infty], \psi_{>}(t \sqrt{(1-\alpha)})$ is bounded below, for $t \geqslant 0$, by a constant (independent of $\alpha$ ) divided by $\sqrt{(1-\alpha)}$. Therefore, we have

$$
\int_{0}^{\infty} \mathrm{d} s . .<\text { const. }(1-\alpha) \int_{0}^{\infty} \mathrm{d} t \exp \left[-t^{2}+2 x t \sqrt{2(1-\alpha)}\right]<\text { const. }(1-\alpha) \exp \left[2(1-\alpha) x^{2}\right]
$$


When $\alpha \rightarrow 1^{-}$, the upper bound (2.25) goes to zero for any given $x$ and thus $\int_{0}^{\infty} \mathrm{d} s \ldots$ vanishes. For $s<0$, $\psi_{<}(s)$ and $\psi_{>}(s)$ are rewritten as

$$
\psi_{<}(s)=\sqrt{2} \exp \left(-s^{2}\right) \int_{-\infty}^{0} \mathrm{~d} v \exp \left[-2 v^{2}+2 v s \sqrt{2}-\phi(v)\right]
$$

and

$$
\psi_{>}(s)=\sqrt{2} \exp \left[\frac{-s^{2}}{(1-\alpha)}\right] \int_{0}^{\infty} \mathrm{d} v \exp \left[-2(1-\alpha) v^{2}+2 v s \sqrt{2}-\phi(v)\right]
$$

When $\alpha \rightarrow 1^{-}$, the integral involved in (2.27) goes to

$$
\int_{0}^{\infty} \mathrm{d} v \exp [2 v s \sqrt{2}-\phi(v)]
$$

which is convergent. Using (2.26), (2.27) and (2.28), we finally obtain

$$
\rho(x)=\frac{1}{\sqrt{2}} g(x) \exp [-\phi(x)] \int_{-\infty}^{0} \mathrm{~d} s \exp (2 x s \sqrt{2})\left[\varphi_{<}(s)+\varphi_{>}(s)\right]^{-1},
$$

with

$$
\left.\begin{array}{l}
\varphi_{<}(s)=\int_{-\infty}^{0} \mathrm{~d} v \exp \left[-2 v^{2}+2 v s \sqrt{2}-\phi(v)\right] \\
\varphi_{>}(s)=\int_{0}^{\infty} \mathrm{d} v \exp [2 v s \sqrt{2}-\phi(v)]
\end{array}\right\}
$$

$g(x)$ is given by (2.20) with $\alpha=1$. For $q_{\mathrm{B}}(x)=0,(2.29)$ reduces to the expression computed by Jancovici [4]. The overall neutrality condition (2.23) is satisfied by (2.29). The dipole of the charge distribution $e\left[\rho(x)-\rho_{\mathrm{B}}(x)\right]$ diverges as suggested by (2.24) with $\alpha=1$. This divergence is related to the slow algebraic decay

$$
\rho(x) \sim \frac{1}{4 x^{2}}
$$

when $x \rightarrow \infty$. We point out that this asymptotic behaviour does not depend on the shape of $\rho_{\mathrm{B}}(x)$ provided that $\rho_{\mathrm{B}}(x)$ decays faster than const./ $x^{2}$. In particular (2.31) is identical to the corresponding behaviour for $q_{\mathrm{B}}(x)=0[4]$.

In figure $1, \rho(x)$ has been drawn for $\alpha=1 / 2, d_{<}=-d_{>}=-1$, and $\rho_{\mathrm{B}}(x)=1-(x+1) / 4$ for $-1 \leqslant x \leqslant 1$. We also recall in this figure the corresponding abrupt step case [4].

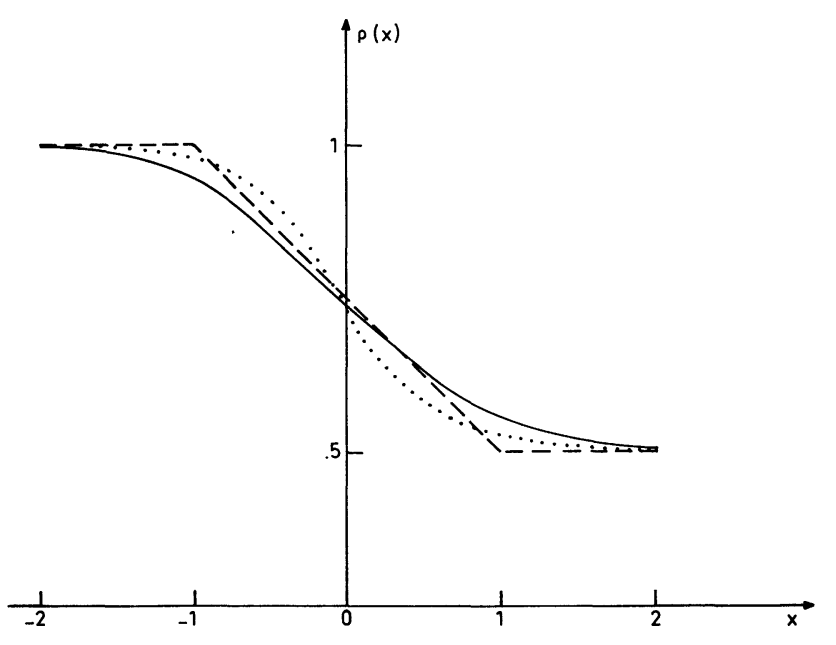

Fig. 1. - The exact particle density $\rho(x)$ at $\Gamma=2$ corresponding to the background density $\rho_{\mathrm{B}}(x)=1$ for $x<-1$, $\rho_{\mathrm{B}}(x)=1-(x+1) / 4$ for $|x| \leqslant 1$, and $\rho_{\mathrm{B}}(x)=1 / 2$ for $x>1\left(\rho(x)\right.$ and $\rho_{\mathrm{B}}(x)$ are in units of $\rho$ and $x$ is in units of $\left.a=1 /(\pi \rho)^{1 / 2}\right)$. Dashed line : $\rho_{\mathrm{B}}(x)$; full line : $\rho(x)$. Dots : the particle density corresponding to $\rho_{\mathrm{B}}(x)=1$ for $x<0$ and $\rho_{\mathrm{B}}(x)=1 / 2$ for $x>0$. 
2.3 THE TRUNCATED TWO-BODY DENSITY. - Let $\rho^{(2, T)}\left(\mathbf{r}_{1}, \mathbf{r}_{2}\right)$ be the truncated (Ursell) two-body distribution function

$$
\rho^{(2, T)}\left(\mathbf{r}_{1}, \mathbf{r}_{2}\right)=\rho^{(2)}\left(\mathbf{r}_{1}, \mathbf{r}_{2}\right)-\rho\left(\mathbf{r}_{1}\right) \rho\left(\mathbf{r}_{2}\right) .
$$

Once the limits $R \rightarrow \infty$ and $N \rightarrow \infty$ have been taken, we have

$$
\rho^{(2, T)}\left(\mathbf{r}_{1}, \mathbf{r}_{2}\right)=-\exp \left[-\beta V_{\mathbf{B}}\left(r_{1}\right)-\beta V_{\mathbf{B}}\left(r_{2}\right)\right]\left|K\left(z_{1} z_{2}^{*}\right)\right|^{2} .
$$

We introduce the Cartesian frame $(x, y)$ in which the origin is at $\mathbf{R}_{0}=R_{0} \hat{\mathbf{r}}_{1}$ and the unit vector along the $x$ direction is $\hat{\mathbf{r}}_{1}$. The corresponding components $\left(x_{1}, 0\right)$ and $\left(x_{2}, y\right)$ of $\mathbf{r}_{1}$ and $\mathbf{r}_{2}$ are kept fixed in the final limit $R_{\min } \rightarrow \infty$. Therefore, the dominant contributions to $K\left(z_{1} z_{2}^{*}\right)$ again come from $p=R_{0}^{2}+R_{0} s \sqrt{2}$. Using (2.16), we find

$$
\begin{aligned}
\rho^{(2, T)}\left(x_{1}, x_{2}, y\right) & =-g\left(x_{1}\right) g\left(x_{2}\right) \exp \left[-\phi\left(x_{1}\right)-\phi\left(x_{2}\right)\right] \times \\
& \times\left|\int_{-\infty}^{\infty} \mathrm{d} s \exp \left[s\left(x_{1}+x_{2}-i y\right) \sqrt{2}\right]\left\{\exp \left(s^{2}\right) \psi_{<}(s)+\exp \left[\frac{s^{2}}{(1-\alpha)}\right] \psi_{>}(s)\right\}^{-1}\right|^{2} .
\end{aligned}
$$

For $q_{\mathrm{B}}(x)=0$, we recover a previous computation [4]. Since the integral $\int_{-\infty}^{\infty} \mathrm{d} s \ldots$ involved in (2.34) can be viewed as the Fourier transform with respect to $y$ of a function of $s$ analytical on the real axis, $\rho^{(2, T)}\left(x_{1}, x_{2}, y\right)$ decays faster than any inverse power of $y$ when $|y| \rightarrow \infty$ ( $x_{1}$ and $x_{2}$ fixed). $x_{1}$ and $y$ being fixed, we find by the method of steepest descent

$$
\rho^{(2, T)}\left(x_{1}, x_{2}, y\right) \sim-g\left(x_{1}\right) \exp \left[x_{1}^{2}-y^{2}-\phi\left(x_{1}\right)\right] \exp \left[-x_{2}^{2}+2 x_{1} x_{2}\right]
$$

when $x_{2} \rightarrow-\infty$ and

$$
\begin{aligned}
\rho^{(2, T)}\left(x_{1}, x_{2}, y\right) \sim-(1-\alpha)^{2} g\left(x_{1}\right) \exp \left[(1-\alpha)\left(x_{1}^{2}-y^{2}\right)+\right. & \left.\phi(\infty)-\phi\left(x_{1}\right)\right] \\
& \times \exp \left[-(1-\alpha) x_{2}^{2}+2(1-\alpha) x_{1} x_{2}\right]
\end{aligned}
$$

when $x_{2} \rightarrow \infty$. We see that the asymptotic behaviours (2.35) and (2.36) are Gaussian. Unlike the one-body density, these behaviours do not depend on the way in which $q_{\mathrm{B}}(x)$ goes to zero at infinity. When $x_{1} \rightarrow-\infty$ and $\infty,(2.35)$ and $(2.36)$ respectively reduce to their well-known bulk forms [15].

Since $\rho^{(2, T)}\left(x_{1}, x_{2}, y\right)$ decays faster than any inverse power of $\left|\mathbf{r}_{2}-\mathbf{r}_{1}\right|$ when particle 2 recedes to infinity, all the multipole moments of $\left[\rho^{(2, T)}\left(x_{1}, x_{2}, y\right)+\pi \rho\left(x_{1}\right) \delta\left(\mathbf{r}_{1}-\mathbf{r}_{2}\right)\right]$ are expected to vanish as shown by the theorems derived in $[12]\left({ }^{2}\right)$. Two of these theorems have been explicitly checked here, namely

$$
\int_{-\infty}^{\infty} \mathrm{d} x_{2} \int_{-\infty}^{\infty} \mathrm{d} y \rho^{(2, \mathrm{~T})}\left(x_{1}, x_{2}, y\right)=-\pi \rho\left(x_{1}\right)
$$

(perfect screening sum rule) and

$$
\int_{-\infty}^{\infty} \mathrm{d} x_{2} \int_{-\infty}^{\infty} \mathrm{d} y\left(\mathbf{r}_{2}-\mathbf{r}_{1}\right) \rho^{(2, T)}\left(x_{1}, x_{2}, y\right)=\dot{0}
$$

Using the same method as in $\S 2.2$ for the one-body density, (2.34) can be extended to the case $\alpha=1$ with the result

$$
\begin{aligned}
\rho^{(2, T)}\left(x_{1}, x_{2}, y\right)=-\frac{1}{2} g\left(x_{1}\right) g\left(x_{2}\right) \exp [ & \left.-\phi\left(x_{1}\right)-\phi\left(x_{2}\right)\right] \times \\
\times & \left|\int_{-\infty}^{0} \mathrm{~d} s \exp \left[s\left(x_{1}+x_{2}-i y\right) \sqrt{2}\right]\left[\varphi_{<}(s)+\varphi_{>}(s)\right]^{-1}\right|^{2} .
\end{aligned}
$$

$\left(^{2}\right)$ In $\left[\rho^{(2, T)}\left(x_{1}, x_{2}, y\right)+\cdots\right]$, the factor $\pi$ appears because of our choice of dimensionless units. 
The asymptotic behaviour of $\int_{-\infty}^{0} \mathrm{~d} s . .$. when $|y| \rightarrow \infty$ is related to the behaviour when $s \rightarrow 0^{-}$of the corresponding integrand in factor of $\exp (-i s y \sqrt{2})$. We easily find by integrations by parts

$$
\rho^{(2, T)}\left(x_{1}, x_{2}, y\right) \sim-g\left(x_{1}\right) g\left(x_{2}\right) \exp \left[2 \phi(\infty)-\phi\left(x_{1}\right)-\phi\left(x_{2}\right)\right] / y^{4}
$$

when $|y| \rightarrow \infty\left(x_{1}\right.$ and $x_{2}$ fixed). When $x_{2} \rightarrow \infty\left(x_{1}\right.$ and $y$ fixed) we obtain by a similar method

$$
\begin{aligned}
\rho^{(2, T)}\left(x_{1}, x_{2}, y\right) & \sim \\
& -g\left(x_{1}\right) \exp \left[\phi(\infty)-\phi\left(x_{1}\right)\right] / x_{2}^{4} .
\end{aligned}
$$

When $x_{2} \rightarrow-\infty\left(x_{1}\right.$ and $y$ fixed $)$, the dominant contributions to $\int_{-\infty}^{0} \mathrm{~d} s .$. come from the large negative| values of $s$, and the asymptotic behaviour of $\rho^{(2, T)}\left(x_{1}, x_{2}, y\right)$ is still given by $(2.35)$ with $\alpha=1$. (2.40) and (2.41) become independent of the shape of $\rho_{\mathrm{B}}(x)$ when $x_{1}$ and $x_{2} \rightarrow \infty$, and $x_{1} \rightarrow \infty$ respectively. These expressions reduce then to those previously computed for $q_{\mathrm{B}}(x)=0$ [4].

Because of the slow algebraic decays (2.40) and (2.41), only the perfect screening (2.37) and dipole (2.38) sum rules are satisfied, in agreement with the theorems derived in [12-13]. The higher-order multipole moments of $\rho^{(2, T)}\left(x_{1}, x_{2}, y\right)$ are not defined. As noticed in the case $q_{\mathrm{B}}(x)=0$ [4], the asymptotic behaviour of the integrated quantity

$$
\int_{-\infty}^{\infty} \mathrm{d} x_{1} \int_{-\infty}^{\infty} \mathrm{d} x_{2} \rho^{(2, T)}\left(x_{1}, x_{2}, y\right)
$$

when $|y| \rightarrow \infty$ cannot be predicted by a general linear response argument [16] because the decay of $\rho^{(2, \dot{T})}\left(x_{1}, x_{2}, y\right)$ when $x_{1}$ or $x_{2} \rightarrow \infty$ is not fast enough.

\section{Local inhomogeneities.}

We consider now the case $\alpha=0$, i.e. $q_{\mathrm{B}}(x)=\rho_{\mathrm{B}}(x)-\rho$ for any $x$. The expressions (2.19) and (2.34) are extended to the cases where $-e q_{\mathrm{B}}(x)$ carries a nonvanishing surface charge. We study the two limits $q_{\mathrm{B}}(x) \rightarrow 0$ (small inhomogeneity) and $\mathrm{d} q_{\mathrm{B}}(x) / \mathrm{d} x \rightarrow 0$ (infinitely smooth inhomogeneity). A few particular cases are considered in detail.

3. 1 THE HOLE WITH ARBITRARY CHARge. - Let $q_{\mathrm{B}}(x)$ be a continuous function with compact support $\left[d_{<}, d_{>}\right]$and $\sigma=\int_{d_{<}}^{d_{>}} \mathrm{d} x q_{\mathrm{B}}(x)$. Let $\rho_{\mathrm{B}, 0}(x)$ be the background shape such as $q_{\mathrm{B}, 0}(x)=q_{\mathrm{B}}(x)$ for $d_{<} \leqslant$ $x \leqslant d_{>}$and $q_{\mathrm{B}, 0}(x)=-\sigma \delta(x+X)$ otherwise $\left(-X<d_{<}\right)$. The total charge carried by $q_{\mathrm{B}, 0}(x)$ is zero so the corresponding one-body density of the particles is obtained from (2.19) with $\alpha=0$. Taking the limit $X \rightarrow \infty, d_{<}, d_{>}$and $x$ being kept fixed, we easily obtain

$$
\begin{aligned}
\rho(x)=\exp \left[-2 x^{2}+\right. & 2 \sigma x-\phi(x)] \times \\
& \times \int_{-\infty}^{\infty} \mathrm{d} s \frac{\exp (2 x s \sqrt{2})}{\varphi(s)},
\end{aligned}
$$

with

$$
\begin{aligned}
\varphi(s)=\sqrt{2} \int_{-\infty}^{\infty} \mathrm{d} v & \exp \left[-2 v^{2}+\right. \\
& +2 \sigma v+2 v s \sqrt{2}-\phi(v)] .
\end{aligned}
$$

The expression (3.1) must be the required particle density corresponding to the distribution $q_{\mathrm{B}}(x)$ alone, by virtue of perfect screening rules. In the limit considered above, we can replace the $\delta$-part of $q_{\mathrm{B}, 0}(x)$ by a continuous function $q_{\mathrm{B}}^{*}(x)$ with compact support $[-X-d,-X]$ and such as

$$
\int_{-X-d}^{-X} \mathrm{~d} x q_{\mathrm{B}}^{*}(x)=-\sigma
$$

We have checked that the corresponding $\rho(x)$ does not depend on the shape of $q_{\mathrm{B}}^{*}(x)$ when $X \rightarrow \infty$ and is indeed given by (3.1). Making the variable changes $t=(s+\sigma / \sqrt{2}-x \sqrt{2}) / \sqrt{2}$ in (3.1) and $u=v-t-x$ in (3.2), we can rewrite (3.1) as

$$
\rho(x)=\exp [-\phi(x)] \int_{-\infty}^{\infty} \mathrm{d} t \frac{\exp \left(-2 t^{2}\right)}{\psi(x+t)}
$$

with

$$
\psi(x+t)=\int_{-\infty}^{\infty} \mathrm{d} u \exp \left[-2 u^{2}-\phi(x+t+u)\right] .
$$

Unlike (3.1), the integral representation (3.4) does not explicitly involve the surface charge $-e \sigma$ carried by $-e q_{\mathrm{B}}(x)$. The expressions (3.4) and (3.5) can obviously be extended to the cases where $d_{<}=-\infty$ and $d_{>}=\infty$, provided that $q_{\mathrm{B}}(x)$ and $x q_{\mathrm{B}}(x)$ are integrable at infinity.

Like in $\S 2$ for the case $0<\alpha<1$, the way in which $\rho(x)$ goes to 1 when $|x| \rightarrow \infty$ depends on the corresponding asymptotic behaviour of $q_{\mathrm{B}}(x)$. In Appendix 1 , we show that (3.4) does satisfy the overall neutrality 
condition

$$
\int_{-\infty}^{\infty} \mathrm{d} x\left[\rho(x)-\rho_{\mathrm{B}}(x)\right]=0
$$

as well as the electrochemical potential balance [11] which becomes here

$$
\int_{-\infty}^{\infty} \mathrm{d} x x\left[\rho(x)-\rho_{\mathrm{B}}(x)\right]=0 .
$$

The truncated two-body distribution function can be computed by starting from (2.34) with $\alpha=0$, and by using the same method as previously for the onebody density. We find

$$
\begin{aligned}
& \rho^{(2, T)}\left(x_{1}, x_{2}, y\right)=- \exp \left[-2\left(x_{2}-x_{1}\right)^{2}+2 i y\left(x_{2}-x_{1}\right)-\phi\left(x_{1}\right)-\phi\left(x_{2}\right)\right] \times \\
& \times \int_{-\infty}^{\infty} \mathrm{d} t_{1} \int_{-\infty}^{\infty} \mathrm{d} t_{2} \exp \left[-2 t_{1}^{2}-2 t_{2}^{2}-2\left(t_{2}-t_{1}\right)\left(x_{2}-x_{1}\right)+\right. \\
&\left.+2 i y\left(t_{2}-t_{1}\right)\right]\left[\psi\left(x_{1}+t_{1}\right) \psi\left(x_{2}+t_{2}\right)\right]^{-1} .
\end{aligned}
$$

When $q_{\mathrm{B}}(x)$ vanishes identically (i.e. $\left.\rho_{\mathrm{B}}(x)=\rho\right), \psi(s)$ reduces to the constant $\sqrt{\pi} / \sqrt{2}$. The integration upon $t_{1}$ and $t_{2}$ in (3.8) is then trivial and we recover the known result [15]

$$
\rho^{(2, T)}\left(x_{1}, x_{2}, y\right)=-\exp \left[-\left(x_{2}-x_{1}\right)^{2}-y^{2}\right]
$$

for the homogeneous system.

Like in the cases $0<\alpha<1$ studied in $\S 2, \rho^{(2, T)}\left(x_{1}, x_{2}, y\right)$ decays faster than any inverse power of $\left|\mathbf{r}_{2}-\mathbf{r}_{1}\right|$ when particle 2 recedes to infinity. All the multipole moments of $\left[\rho^{(2, T)}\left(x_{1}, x_{2}, y\right)+\pi \rho\left(x_{1}\right) \delta\left(\mathbf{r}_{2}-\mathbf{r}_{1}\right)\right]$ are again expected to vanish. We have explicitly checked that the perfect screening (2.37) and dipole (2.38) sum rules are indeed satified by (3.8).

3.2 LiNEAR RESPONSE THEORY AND MACROSCOPIC PERFECT SCREENING. - Now we study the limit of (3.4) when $q_{\mathrm{B}}(x)$ goes to zero everywhere. Writing

$$
\left.\begin{array}{rl}
\exp [-\phi(x)] & =1-\phi(x)+\cdots, \\
\psi(x+t) & =\sqrt{\frac{\pi}{2}}\left[1-\sqrt{\frac{2}{\pi}} \int_{-\infty}^{\infty} \mathrm{d} u \exp \left(-2 u^{2}\right) \phi(x+t+u)+\cdots\right],
\end{array}\right\}
$$

we obtain up to the first order in $\phi$

$$
\rho(x)-1=-\phi(x)+\frac{2}{\pi} \int_{-\infty}^{\infty} \mathrm{d} t \int_{-\infty}^{\infty} \mathrm{d} u \exp \left(-2 t^{2}-2 u^{2}\right) \phi(x+t+u) .
$$

Making the variable changes $\left(x_{2}=x+t+u, y=t-u\right)$, we can rewrite (3.11) as

$$
\rho(x)-1=-\phi(x)+\frac{1}{\pi} \int_{-\infty}^{\infty} \mathrm{d} x_{2} \int_{-\infty}^{\infty} \mathrm{d} y \exp \left[-\left(x_{2}-x\right)^{2}-y^{2}\right] \phi\left(x_{2}\right) .
$$

The exponential involved in (3.12) is nothing but minus the truncated two-body distribution function of the homogeneous system (3.9). Therefore, (3.12) is identical to the expression given by the linear response theory, as it should.

Let us consider an infinitely smooth background and study the corresponding particle density. That is, we put $q_{\mathrm{B}}(x)=f(x / L)$ where $f(\xi)$ is a given function and we compute the asymptotic form of (3.4) when $L \rightarrow \infty$. $\phi(x)$ takes the form

$$
\phi(x)=L^{2} F(x / L)
$$

where $F(\xi)$ is such as

$$
\frac{\mathrm{d}^{2} F}{\mathrm{~d} \xi^{2}}(\xi)=4 f(\xi)
$$

Making the variable changes $\xi_{u}=u / L$ in (3.5) and $\xi_{t}=t / L$ in (3.4), we get $\left(\xi_{x}=x / L\right)$

$$
\rho(x)=\exp \left[-L^{2} F\left(\xi_{x}\right)\right] \int_{-\infty}^{\infty} \mathrm{d} \xi_{t} \exp \left(-2 L^{2} \xi_{t}^{2}\right) / \psi_{L}\left(\xi_{x}+\xi_{t}\right)
$$


with

$$
\psi_{L}\left(\xi_{x}+\xi_{t}\right)=\int_{-\infty}^{\infty} \mathrm{d} \xi_{u} \exp \left[-2 L^{2} \xi_{u}^{2}-L^{2} F\left(\xi_{x}+\xi_{t}+\xi_{u}\right)\right]
$$

In the large- $L$ limit, $\psi_{L}\left(\xi_{x}+\xi_{t}\right)$ can be computed by the method of steepest descent around the point $\xi_{\mathrm{D}}$ such as

$$
4 \xi_{\mathrm{D}}+\frac{\mathrm{d} F}{\mathrm{~d} \xi}\left(\xi_{x}+\xi_{t}+\xi_{\mathrm{D}}\right)=0
$$

with the result

$$
\psi_{L}\left(\xi_{x}+\xi_{t}\right) \sim \sqrt{\pi} \exp \left[-2 L^{2} \xi_{\mathrm{D}}^{2}-L^{2} F\left(\xi_{x}+\xi_{t}+\xi_{\mathrm{D}}\right)\right] / L \sqrt{2\left[1+f\left(\xi_{x}+\xi_{t}+\xi_{\mathrm{D}}\right)\right]} .
$$

Inserting (3.18) in (3.15), we have

$$
\begin{gathered}
\rho(x) \sim L \sqrt{\frac{2}{\pi}} \exp \left[-L^{2} F\left(\xi_{x}\right)\right] \int_{-\infty}^{\infty} \mathrm{d} \xi_{t} \sqrt{\left[1+f\left(\xi_{x}+\xi_{t}+\xi_{\mathrm{D}}\right)\right]} \times \\
\times \exp \left[-2 L^{2} \xi_{t}^{2}+2 L^{2} \xi_{\mathrm{D}}^{2}+L^{2} F\left(\xi_{x}+\xi_{t}+\xi_{\mathrm{D}}\right)\right] .
\end{gathered}
$$

$\sqrt{[1+\cdots]}$ is a slowly-varying function of $\xi_{t}$ compared to the exponential involved in $\int_{-\infty}^{\infty} \mathrm{d} \xi_{t} \ldots$. The latter integral can then be computed by the method of steepest descent around the point $\xi_{\mathrm{N}}$ such as

$$
-4 \xi_{\mathrm{N}}+\frac{\mathrm{d} F}{\mathrm{~d} \xi}\left(\xi_{x}+\xi_{\mathrm{N}}+\xi_{\mathrm{D}}\right)+\left.\left[4 \xi_{\mathrm{D}}+\frac{\mathrm{d} F}{\mathrm{~d} \xi}\left(\xi_{x}+\xi_{\mathrm{N}}+\xi_{\mathrm{D}}\right)\right] \frac{\partial \xi_{\mathrm{D}}}{\partial \xi_{t}}\right|_{\xi_{t}=\xi_{\mathrm{N}}}=0
$$

which can be rewritten using (3.17) as

$$
\xi_{\mathrm{N}}+\xi_{\mathrm{D}}\left(\xi_{x}, \xi_{\mathrm{N}}\right)=0
$$

The second derivative with respect to $\xi_{t}$ at $\xi_{t}=\xi_{\mathrm{N}}$ of the argument of the exponential involved in (3.19) is found to be

$$
-4 L^{2} /\left[1+f\left(\xi_{x}\right)\right]
$$

where we have combined identity (3.21) and the differentiation with respect to $\xi_{t}$ of equation (3.17). After these manipulations, (3.19) finally reduces to

$$
\rho(x) \sim 1+f\left(\xi_{x}\right)=\rho_{\mathrm{B}}(x)
$$

when $L \rightarrow \infty$. (3.23) shows that the background is perfectly screened at each point by the particles : the system does not accept a non-vanishing macroscopic charge distribution. This result is in complete agreement with the predictions of macroscopic electrostatics applied to a conducting medium in equilibrium.

3.3 Particular CaSes. - We consider the cases where $q_{\mathrm{B}}(x)=\delta(-1 \leqslant \delta)$ for $|x| \leqslant l / 2$ and $q_{\mathrm{B}}(x)=0$ otherwise. $\phi(x)$ is then given by

$$
\left.\begin{array}{lll}
\phi(x)=2 \delta x^{2} & \text { for } & |x| \leqslant l / 2, \\
\phi(x)=-\frac{\delta l^{2}}{2}+2 \delta l|x| & \text { for } & l / 2 \leqslant|x| .
\end{array}\right\}
$$

Inserting (3.24) in (3.5), we find

$$
\begin{array}{r}
\rho(x)=\frac{2 \sqrt{2}}{\sqrt{\pi}} \exp \left[-\frac{\delta l^{2}(1+\delta)}{2}-\phi(x)\right] \int_{-\infty}^{\infty} \mathrm{d} s \exp \left[-2(s-x)^{2}\right]\{\exp (2 \delta l s)[1-\operatorname{Erf}((s+\gamma) \sqrt{2})]+ \\
+\exp (-2 \delta l s)[1+\operatorname{Erf}((s-\gamma) \sqrt{2})]+(1+\delta)^{-1 / 2} \exp \left[-\frac{\delta l^{2}(1+\delta)}{2}-\frac{2 \delta s^{2}}{(1+\delta)}\right] \times \\
\left.\times\left[\operatorname{Erf}\left((s+\gamma) \sqrt{\frac{2}{(1+\delta)}}\right)-\operatorname{Erf}\left((s-\gamma) \sqrt{\frac{2}{(1+\delta)}}\right)\right]\right\}^{-1},
\end{array}
$$


where $\gamma=l(1+\delta) / 2$ and Erf is the error function,

$$
\operatorname{Erf}(z)=\frac{2}{\sqrt{\pi}} \int_{0}^{z} \mathrm{~d} \xi \exp \left(-\xi^{2}\right)
$$

In the respective limits, $\left(\delta \rightarrow \infty, l \rightarrow 0, \sigma=\delta l\right.$ fixed) and $\left(\delta \rightarrow-1^{+}, l\right.$ fixed $), \rho(x)$ takes a slightly more compact form than (3.25), namely

$$
\begin{gathered}
\rho(x)=\frac{2 \sqrt{2}}{\sqrt{\pi}} \exp \left(-\frac{\sigma^{2}}{2}-2 \sigma|x|\right) \int_{-\infty}^{\infty} \mathrm{d} s \exp \left[-2(s-x)^{2}\right] \times \\
\times\left\{\exp (2 \sigma s)\left[1-\operatorname{Erf}\left(\left(s+\frac{\sigma}{2}\right) \sqrt{2}\right)\right]+\exp (-2 \sigma s)\left[1+\operatorname{Erf}\left(\left(s-\frac{\sigma}{2}\right) \sqrt{2}\right)\right]\right\}^{-1}
\end{gathered}
$$

and

$$
\begin{aligned}
\rho(x)=\frac{2 \sqrt{2}}{\sqrt{\pi}} \exp [-\phi(x)] \int_{-\infty}^{\infty} & \mathrm{d} s \exp \left[-2(s-x)^{2}\right] \times \\
\times & \left\{\exp (2 l s)\left[1+\operatorname{Erf}(s \sqrt{2})+\frac{\exp \left(-2 s^{2}\right)}{s \sqrt{2 \pi}}\right]+\right. \\
+ & \left.\exp (-2 l s)\left[1-\operatorname{Erf}(s \sqrt{2})-\frac{\exp \left(-2 s^{2}\right)}{s \sqrt{2 \pi}}\right]\right\}^{-1} .
\end{aligned}
$$

In figure 2, we have drawn $\rho(x)$ for $\delta=1$ and $l=1$.

When $l \rightarrow \infty, \delta$ and $x-l / 2$ being kept fixed, we recover the abrupt step case [4]. When $\delta \rightarrow \infty, l$ and $x$ fixed, we find

$$
\begin{gathered}
\rho(x)=\infty \quad \text { for } \quad 0 \leqslant x \leqslant l / 2 \\
\rho(x)=2 \sqrt{\frac{2}{\pi}} \int_{-\infty}^{\infty} \mathrm{d} \xi \exp \left[-2\left(\xi+\frac{l}{2}-x\right)^{2}\right] /[1+\operatorname{Erf}(\xi \sqrt{2})] \text { for } l / 2<x
\end{gathered}
$$

$(\rho(-x)=\rho(x))$. For $x>l / 2,(3.29)$ is identical to the density of particles near an impenetrable wall carrying an infinite negative surface charge [1]. When $x \rightarrow(l / 2)^{+}, \rho(x)$ diverges like

$$
\rho(x) \sim \frac{1}{4\left(x-\frac{l}{2}\right)^{2}},
$$

which is not integrable at $x=l / 2$.

\section{The oscillating background.}

We show that the integral representations (3.4) and (3.8) remain valid when $\rho_{\mathrm{B}}(x)$ is a periodic function with period $\lambda$. For a suitable choice of $\rho_{\mathrm{B}}(x)$, all the Fourier components of $\rho(x)$ can be explicitly computed.

4.1 THE ONE- AND TWO-BODY DENSITIES. - For any given periodic function $\rho_{\mathrm{B}}(x)$, we define the mean density

$$
\rho=\frac{1}{\lambda} \int_{0}^{\lambda} \mathrm{d} x \rho_{\mathrm{B}}(x)
$$

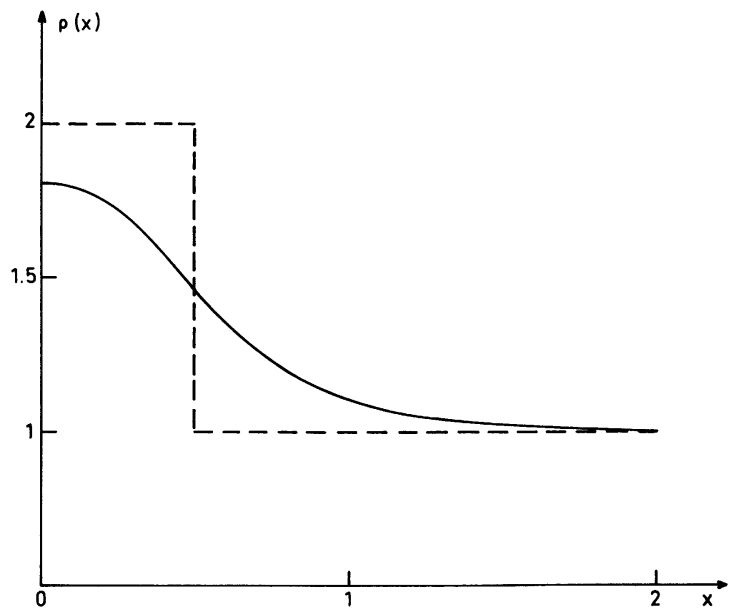

Fig. 2. - The same as figure 1 for $\rho_{\mathrm{B}}(x)=1$ for $|x|>1 / 2$ and $\rho_{\mathrm{B}}(x)=2$ for $|x| \leqslant 1 / 2(\rho(-x)=\rho(x))$.

furthermore there is at least one choice of the origin on the $x$-axis for which

$$
\int_{0}^{\lambda} \mathrm{d} x x q_{\mathrm{B}}^{\prime}(x)=0
$$


with $q_{\mathrm{B}}(x)=\rho_{\mathrm{B}}(x)-\rho, \rho$ being given by (4.1). Let $\rho_{\mathrm{B}, M}(x)$ be the background shape such as

$$
\begin{array}{ll}
\rho_{\mathbf{B}, M}(x)=\rho_{\mathbf{B}}(x) & \text { for } \quad|x| \leqslant M \lambda, \\
\rho_{\mathbf{B}, M}(x)=\rho & \text { for } \quad M \lambda<|x|,
\end{array}
$$

where $M$ is a positive integer. The particle density $\rho_{M}(x)$ (in units of the $\rho$ given by (4.1)) corresponding to $\rho_{\mathrm{B}, M}(x)$ is given by (3.4) and (3.5) with $\phi(x)$ replaced by the electrostatic potential $\phi_{M}(x)$ created by the charge distribution $-e q_{\mathrm{B}, M}(x)=-e\left[\rho_{\mathrm{B}, M}(x)-\rho\right]$. Using (4.1) and (4.2), we find for $x=n \hat{\lambda}+\xi$ with $-M \leqslant n<M$ and $0 \leqslant \xi<\lambda$

$$
\phi_{M}(x)=\phi(x)=4 \int_{0}^{\xi} \mathrm{d} \xi^{\prime}\left(\xi-\xi^{\prime}\right) q_{\mathrm{B}}\left(\xi^{\prime}\right)
$$

(we have chosen the origin of the electrostatic potentials at $x=0$ ). Since the total surface charge carried by $-e q_{\mathrm{B}, M}(x)$ is zero, we have also

$$
\phi_{M}(x)=0 \text { for } M \lambda \leqslant|x| .
$$

Taking now the limit $M \rightarrow \infty, x$ being kept fixed, we see that $\rho(x)=\lim _{M \rightarrow \infty} \rho_{M}(x)$ is obviously still given by (3.4), with $\phi(x)$ computed from (4.4). Similarly, the integral representation (3.8) of the truncated two-body density remains also valid in the present periodic case.

Using

$$
\phi(x+\lambda)=\phi(x),
$$

we immediately see that $\rho(x)$ is a periodic function with period $\lambda$. In Appendix 2, we show that

$$
\int_{0}^{\lambda} \mathrm{d} x\left[\rho(x)-\rho_{\mathrm{B}}(x)\right]=0
$$

which is a trivial consequence of the overall neutrality.

Using (4.6), we easily check that $\rho^{(2, T)}\left(x_{1}, x_{2}, y\right)$ has the expected periodicity

$$
\rho^{(2, T)}\left(x_{1}+\lambda, x_{2}+\lambda, y\right)=\rho^{(2, T)}\left(x_{1}, x_{2}, y\right) .
$$

$\rho^{(2, T)}\left(x_{1}, x_{2}, y\right)$ decays again faster than any inverse power of $\left|\mathbf{r}_{2}-\mathbf{r}_{1}\right|$ when particle 2 recedes to infinity, and thus satisfies multipole sum rules [12] like in $\S 3$, in particular (2.37) and (2.38).

4.2 Particular CaSes. - Let us consider the cases where

$$
q_{\mathrm{B}}(x)=\frac{k^{2} \delta}{4} \frac{(\delta+\cos k x)}{(1+\delta \cos k x)^{2}}
$$

with $|\delta|<1$ and $k=2 \pi / \lambda . q_{\mathrm{B}}(x)$ can be rewritten as

$$
q_{\mathrm{B}}(x)=-\frac{1}{4} \frac{\mathrm{d}^{2}}{\mathrm{~d} x^{2}} \ln (1+\delta \cos k x)
$$

Using (4.10) in (4.4), we obtain

$$
\phi(x)=-\ln \frac{(1+\delta \cos k x)}{(1+\delta)} .
$$

Taking advantage of the simple form of the Boltzmann factor $\exp [-\phi(x)]$, we find from (3.4)

$$
\rho(x)=\sqrt{\frac{2}{\pi}}(1+\delta \cos k x) \int_{-\infty}^{\infty} \mathrm{d} s \frac{\exp \left[-2(s-x)^{2}\right]}{(1+\varepsilon \cos k s)}
$$

with $\varepsilon=\delta \exp \left(-k^{2} / 8\right)$. The Fourier components of $\rho(x)$ can be computed explicitly, namely

$$
\rho(x)=1+\sum_{n=1}^{\infty} a_{n} \cos (n k x),
$$

with

$$
\begin{aligned}
a_{n}= & \frac{\exp \left(-n^{2} k^{2} / 8\right)}{\sqrt{\left(1-\varepsilon^{2}\right)}}\left[\frac{\left(\sqrt{\left(1-\varepsilon^{2}\right)}-1\right)}{\varepsilon}\right]^{n} \times \\
& \times\left\{2+\varepsilon\left[\exp \left(-n k^{2} / 4\right)\left(\frac{\sqrt{\left(1-\varepsilon^{2}\right)}-1}{\varepsilon}\right)+\right.\right. \\
& \left.\left.+\exp \left(n k^{2} / 4\right)\left(\frac{\varepsilon}{\sqrt{\left(1-\varepsilon^{2}\right)}-1}\right)\right]\right\}
\end{aligned}
$$

On the other hand, the Fourier series of $\rho_{\mathrm{B}}(x)$ reads

$$
\rho_{\mathrm{B}}(x)=1+\sum_{n=1}^{\infty} a_{n, \mathrm{~B}} \cos (n k x),
$$

with

$$
\begin{aligned}
a_{n, \mathrm{~B}} & =\frac{n \delta k^{2}}{4 \sqrt{\left(1-\delta^{2}\right)}}\left[\frac{\left(\sqrt{\left(1-\delta^{2}\right)}-1\right)}{\delta}\right]^{n} \times \\
\times & {\left[\frac{\delta}{\left(\sqrt{\left(1-\delta^{2}\right)}-1\right)}-\frac{\left(\sqrt{\left(1-\delta^{2}\right)}-1\right)}{\delta}\right] . }
\end{aligned}
$$

The Fourier series (4.13) obviously satisfies the identity (4.7).

When $\delta \rightarrow 0$ ( $k$ fixed), $q_{\mathrm{B}}(x)$ takes the simpler form

$$
q_{\mathrm{B}}(x)=\frac{k^{2} \delta}{4} \cos k x
$$

up to the first order in $\delta$. All the coefficients $a_{n}$ with $n \geqslant 2$ are at least of order $\delta^{2}$, and thus we have

$$
\rho(x)-1 \sim \delta\left[1-\exp \left(-k^{2} / 4\right)\right] \cos k x
$$

when $\delta \rightarrow 0 .(\rho(x)-1)$ becomes proportional to $q_{\mathrm{B}}(x)$ with the proportionality constant

$$
4\left[1-\exp \left(-k^{2} / 4\right)\right] / k^{2}
$$

which is indeed the expression given by the linear response theory. 
When $k \rightarrow 0$ ( $\delta$ fixed), we find for any $n \geqslant 1$

$$
\frac{a_{n}}{a_{n, \mathrm{~B}}} \rightarrow 1 \text {. }
$$

(4.19) implies that $[\rho(x)-1] /\left[\rho_{\mathrm{B}}(x)-1\right]$ goes to 1 , in agreement with the general property of local screening discussed in $\S 3.2$.

For fixed values of $k$ and $\delta, a_{n}$ decays essentially like a Gaussian when $n \rightarrow \infty$, whereas $a_{n, \mathrm{~B}}$ decays only like an exponential. In particular, when $k \rightarrow \infty$ ( $\delta$ fixed), the ratio $a_{n+1, \mathrm{~B}} / a_{n, \mathrm{~B}}$ remains obviously finite whereas $a_{n+1} / a_{n}$ goes to zero like

$$
-\frac{\delta}{2} \exp \left(-n k^{2} / 4\right)
$$

Only the first harmonic $a_{1}$ does not vanish and $\rho(x)$ reduces to the very simple form

$$
\rho(x) \sim 1+\delta \cos k x
$$

when $k \rightarrow \infty$. Note that (4.21) is proportional to the Boltzmann factor $\exp [-\phi(x)]$ : the particles behave as if they were free (without correlations) because the period of the imposed oscillations becomes small compared to the correlation length.

In figure 3 , we have drawn up $\rho(x)$ for $\delta=1 / 2$ and $k=2(\lambda=\pi)$. The Fourier series (4.13) is very convenient for practically computing $\rho(x)$ because of the very fast decay of $a_{n}$ when $n$ increases.

\section{Non-Coulomb external potential.}

The previous integral representations can be extended to the cases where the particles are also submitted to an arbitrary external potential $V_{\text {ext }}(x)$ (in units of $k_{\mathrm{B}} T$ ) depending on the $x$-direction only; this potential is not necessarily created by external charges and may take into account impenetrable walls for instance. For that purpose, we again start from the finite system with circular symmetry described in $\S 2$. In addition to the Coulomb potential created by the background, an external potential $V_{\text {ext }}(r)$ is applied to the particles. $V_{\text {ext }}(r)$ has the radial symmetry and vanishes everywhere except in a finite neighbourhood of $R_{\min }$. Therefore, the technique used in $\S 2$ can be applied to the present case. That immediately leads to integral representations of the one- and two-body densities which are similar to (2.19) and (2.34), with $\phi+V_{\text {ext }}$ in place of $\phi$ alone. This simple substitution of $\phi$ by $\phi+V_{\text {ext }}$ is obviously still valid for the expressions derived in $\S 3$ and 4 .

The present generalization allows us to consider a large variety of models. Here, we restrict ourselves to briefly mentioning how to recover some known results which have been derived by different methods.

Let us consider the abrupt (i.e. $q_{\mathrm{B}}(x)=0$ ) step background described in $\S 2$. Putting $V_{\text {ext }}(x)=$ const. $=V_{0}$ for $x<0$ and $V_{\text {ext }}(x)=0$ for $x>0$, we recover the results derived recently by Blum and Rosinberg [6]

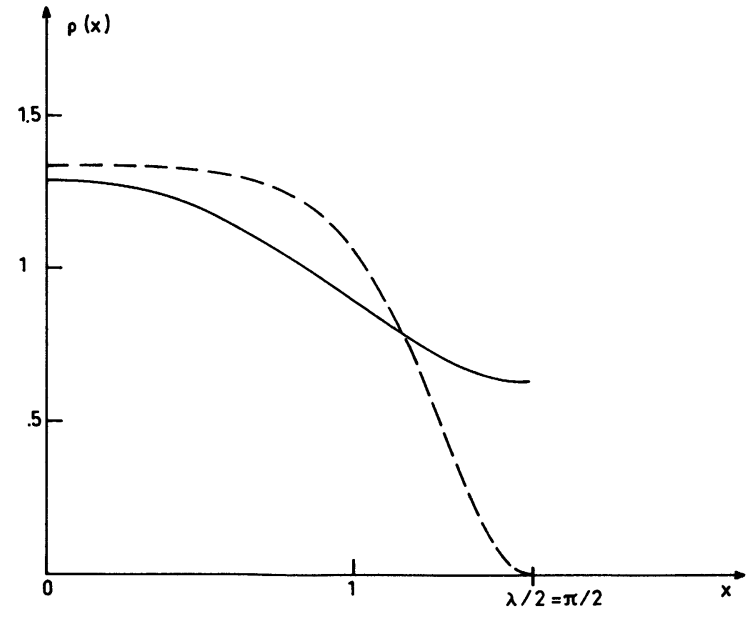

Fig. 3. - The same as figure 1 for $\rho_{\mathrm{B}}(x)=1+(1+2 \cos 2 x) /$ $(2+\cos 2 x)^{2}(\rho(\pi-x)=\rho(x))$.

for an ideally polarizable interface. In their formulation, an impermeable membrane is introduced at $x=0$ and separates the "left " region $(x<0)$ from the "right" region $(x>0)$. Furthermore, they work in the Grand Canonical Ensemble and impose different fugacities to the « left " and " right " particles. The ratio of these fugacities is just $\exp \left(-V_{0}\right)$.

In the case of the square hole described in $\S 3.3$, we set $\delta=-1$ and add the external potential $V_{\text {ext }}(x)=\infty$ for $|x|<l / 2$ and $V_{\text {ext }}(x)=0$ for $|x| \geqslant$ $l / R$. Taking then the limit $l \rightarrow \infty,(x-l / 2)$ being kept fixed and positive, we recover the expressions computed by Jancovici [1] near an impenetrable insulating wall.

Finally, let $\rho_{\mathrm{B}}(x)$ and $V_{\text {ext }}(x)$ be $\rho_{\mathrm{B}}(x)=1$ and $V_{\text {ext }}(x)=V_{0}+(\ln (1+\delta)) / 2$ for $l / 2+d \leqslant|x|$, $\rho_{\mathrm{B}}(x)=0$ and $V_{\text {ext }}(x)=\infty$ for $l / 2<|x|<l / 2+d$, and $\rho_{\mathrm{B}}(x)=1+\delta$ and $V_{\text {ext }}(x)=0$ for $|x| \leqslant l / 2$. When $\delta \rightarrow \infty$, the correlation length of the inner region $(|x| \leqslant l / 2)$ goes to zero and the excess charges stand close to the walls surrounding this region. The latter is then expected to behave as a perfect conductor [17]. In this infinite- $\delta$ limit, we have checked that our $\rho(x)$ (for $|x| \geqslant l / 2+d$ ) indeed reduces to the particle density computed by Forrester [18] near a conducting wall $\left({ }^{3}\right)$. The derivation of Forrester explicitly takes into account the image forces induced by the conductor and thus involves technical methods which are very different from ours. However, in our approach, the electrostatic potential difference $(\Phi(\infty)-\Phi(l / 2))$ turns out to be $\left(-V_{0}+\ln 2-1 / 2\right)$ rather than $-V_{0}$.

$\left({ }^{3}\right)$ The one-body density found by our limit procedure does not depend on the width $l$ of the perfect conductor. The " plasma " regions $(x \leqslant-l / 2-d)$ and $(x \geqslant l / 2+d)$ are completely uncorrelated : the electric field created by a charge belonging to the region $(x \leqslant-l / 2-d)$ is completely screened in the opposite region $(x \geqslant l / 2+d)$ by the own image of that charge. 
A simple scaling argument shows that $(\Phi(l / 2)-\Phi(0))$ reduces precisely to $(1 / 2-\ln 2)$ and thus $(\Phi(\infty)-$ $\Phi(0))$ is indeed equal to $-V_{0}$ as it should. As a consequence, the inner region $(|x| \leqslant l / 2)$ behaves as a perfect conductor only up to a constant potential difference across its surface.

\section{Conclusion.}

We have studied plane charged interfaces of the twodimensional jellium at $\Gamma=2$. We have expressed the one- and two-body densities of the particles as explicit functionals of the electrostatic potential induced by the background and of the external potential applied to the particles. Most previous exact results [1-2, 4-6] can be recovered by taking suitable limits in our general expressions; in particular, surfaces involving impenetrable walls are described by letting $V_{\text {ext }}(x)$ go to $\infty$ outside the "plasma" region.

Standard sum rules established for inhomogeneous charged systems have been explicitly checked. For the one-body density, the overall neutrality condition and the electrochemical potential balance [11] are satisfied. The conditional multipole sum rules [12] for the twobody density are also satisfied by our expressions. In most cases, the two-body correlations decay faster than any power law when one of the particles recedes to infinity; all the multipole moments of the chargecharge correlation function should then vanish. When the background fills only one half-space (i.e. $\rho_{\mathrm{B}}(x)=0$ for $x>0$ ) long-range correlations appear in the $y$ direction : near the step, a positive charge cannot be "strongly" screened because of the absence of negative charges in the whole region $(x>0)$. In this case, only the first multipole moments of the charge-charge correlation function vanish, in agreement with the theorems derived in $[12,13]$; if there is also an impenetrable wall located at $x=0$, the asymptotic form of this correlation function can be predicted by general arguments $[13,14,16]$.

As illustrated by the previous sum rules, the present model exhibits the purely Coulomb characteristics of electrified interfaces. Then, it can be used for essentially two purposes. First, it provides a test bench for approximate theories. Second, by adjusting the background shape and the external potential, one can " simulate " a large variety of real charged surfaces : electrolytes near a conducting wall, metallic surfaces, permeable or impermeable membranes, ideally polarizable interfaces, etc.

\section{Acknowledgments.}

The authors acknowledge with thanks B. Jancovici for critical reading of the manuscript and for helpful discussions and suggestions, and P. J. Forrester for having made unpublished results available to them. This work was supported in part by AFOSR grant $n^{\circ} 82-0016$.

\section{Appendix 1}

In this Appendix, we show that the integral representations (2.19) and (3.4) of $\rho(x)$ satisfy the sum rules (2.23), (2.24) and (3.6), (3.7) respectively.

Let us first consider the smooth step case where $\rho(x)$ is given by $(2.19)$. We define

$$
Q=\int_{-\infty}^{\infty} \mathrm{d} x\left[\rho(x)-\rho_{\mathrm{B}}(x)\right] .
$$

Using (2.22), we rewrite $Q$ as

$$
Q=Q_{<}+Q_{>}
$$

with

$$
Q_{<}=\int_{-\infty}^{0} \mathrm{~d} x[\rho(x)-1]
$$

and

$$
Q_{>}=\int_{0}^{\infty} \mathrm{d} x[\rho(x),-(1-\alpha)]
$$

Replacing 1 by

$$
\frac{1}{\sqrt{\pi}} \int_{-\infty}^{\infty} \mathrm{d} s \exp \left[-(s-x \sqrt{2})^{2}\right]
$$

in (A1.3) and $(1-\alpha)$ by

$$
\frac{\sqrt{(1-\alpha)}}{\sqrt{\pi}} \int_{-\infty}^{\infty} \mathrm{d} s \exp \left\{-\left[\frac{s}{\sqrt{(1-\alpha)}}-x \sqrt{2(1-\alpha)}\right]^{2}\right\}
$$


in (A1.4), we obtain after integrating upon $x$

$$
Q_{<}=\frac{1}{\sqrt{2}} \int_{-\infty}^{\infty} \mathrm{d} s\left\{\frac{\exp \left(s^{2}\right) \psi_{<}(s)}{\left[\exp \left(s^{2}\right) \psi_{<}(s)+\exp \left(s^{2} /(1-\alpha)\right) \psi_{>}(s)\right]}-\frac{1}{2}[1-\operatorname{Erf}(s)]\right\}
$$

and

$$
Q_{>}=\frac{1}{\sqrt{2}} \int_{-\infty}^{\infty} \mathrm{d} s\left\{\frac{\exp \left(s^{2} /(1-\alpha)\right) \psi_{>}(s)}{\left[\exp \left(s^{2}\right) \psi_{<}(s)+\exp \left(s^{2} /(1-\alpha)\right) \psi_{>}(s)\right]}-\frac{1}{2}[1+\operatorname{Erf}(\dot{s} / \sqrt{(1-\alpha)})]\right\} .
$$

Adding (A1.7) to (A1.8), and using (A1.1) and (A1.2), we find

$$
\int_{-\infty}^{\infty} \mathrm{d} x\left[\rho(x)-\rho_{\mathrm{B}}(x)\right]=\frac{1}{2 \sqrt{2}} \int_{-\infty}^{\infty} \mathrm{d} s[\operatorname{Erf}(s)-\operatorname{Erf}(s / \sqrt{(1-\alpha)})]
$$

which clearly vanishes for parity reasons.

Let $\mathfrak{D}$ be

$$
\mathfrak{D}=\int_{-\infty}^{\infty} \mathrm{d} x x\left[\rho(x)-\rho_{\mathrm{B}}(x)\right]
$$

D can be rewritten as

$$
\mathfrak{D}=\mathfrak{D}_{<}+\mathfrak{D}_{>}+\frac{\phi(\infty)}{4}
$$

with

and

$$
\mathfrak{D}_{<}=\int_{-\infty}^{0} \mathrm{~d} x x[\rho(x)-1]
$$

$$
D_{>}=\int_{0}^{\infty} \mathrm{d} x x[\rho(x)-(1-\alpha)]
$$

Replacing again 1 by (A1.5) in (A1.12) and (1- $-\alpha$ by (A1.6) in (A1.13), we obtain similarly to (A1.7, A1.8)

$$
\begin{aligned}
\mathfrak{D}_{<}=\frac{1}{4} \int_{-\infty}^{\infty} \mathrm{d} s\left\{\frac{1}{\left[\exp \left(s^{2}\right) \psi_{<}(s)+\exp \left(s^{2} /(1-\alpha)\right) \psi_{>}(s)\right]} \frac{\mathrm{d}}{\mathrm{d} s}\left[\exp \left(s^{2}\right) \psi_{<}(s)\right]+\right. \\
\left.\quad+\frac{\exp \left(-s^{2}\right)}{\sqrt{\pi}}-s[1-\operatorname{Erf}(s)]\right\}
\end{aligned}
$$

and

$$
\begin{aligned}
\mathfrak{D}_{>}=\frac{1}{4} \int_{-\infty}^{\infty} \mathrm{d} s\left\{\frac{1}{\left[\exp \left(s^{2}\right) \psi_{<}(s)+\exp \left(s^{2} /(1-\alpha)\right) \psi_{>}(s)\right]} \frac{\mathrm{d}}{\mathrm{d} s}\left[\exp \left(s^{2} /(1-\alpha)\right) \psi_{>}(s)\right]\right. \\
\left.-\frac{\exp \left(-s^{2} /(1-\alpha)\right)}{\sqrt{\pi(1-\alpha)}}-\frac{s}{(1-\alpha)}[1+\operatorname{Erf}(s / \sqrt{(1-\alpha)})]\right\} .
\end{aligned}
$$

Rewriting the integrals upon $s$ involved in (A1.14) and (A1.15) as $\lim _{T \rightarrow \infty} \int_{-T}^{T} \mathrm{~d} s \ldots$, we get from (A1.11), (A1.14) and (A1.15)

$$
\mathfrak{D}=\frac{1}{4} \lim _{T \rightarrow \infty}\left\{\frac{\alpha T^{2}}{(\alpha-1)}+\ln \left[\frac{\exp \left(T^{2}\right) \psi_{<}(T)+\exp \left(T^{2} /(1-\alpha)\right) \psi_{>}(T)}{\exp \left(T^{2}\right) \psi_{<}(-T)+\exp \left(T^{2} /(1-\alpha)\right) \psi_{>}(-T)}\right]\right\}+\frac{\phi(\infty)}{4} .
$$


From the definitions (2.21) we easily obtain when $T \rightarrow \infty$

$$
\begin{aligned}
& \psi_{<}(-T) \sim \sqrt{\pi} \\
& \psi_{<}(T) \quad \sim \frac{\exp [-\phi(0)]}{2 T} \exp \left(-T^{2}\right), \\
& \psi_{>}(-T) \sim \frac{\exp [-\phi(0)]}{2 T} \exp \left(-T^{2} /(1-\alpha)\right), \\
& \psi_{>}(T) \sim \frac{\sqrt{\pi}}{\sqrt{(1-\alpha)}} \exp [-\phi(\infty)] .
\end{aligned}
$$

Inserting these asymptotic behaviours in (A1.16) and using the definition (A1.10) of D, we finally obtain

$$
\int_{-\infty}^{\infty} \mathrm{d} x x\left[\rho(x)-\rho_{\mathrm{B}}(x)\right]=-\frac{1}{8} \ln (1-\alpha)
$$

which is the required result.

We turn now to the hole case where $\rho(x)$ is given by (3.4). For the present derivations, it is more convenient to rewrite (3.4) as

$$
\rho(x)=\exp [-\phi(x)] \int_{-\infty}^{\infty} \mathrm{d} s \frac{\exp \left[-2(s-x)^{2}\right]}{\psi(s)}
$$

with

$$
\psi(s)=\int_{-\infty}^{\infty} \mathrm{d} v \exp \left[-2(v-s)^{2}-\phi(v)\right]
$$

$Q$ is again defined by (A1.1). $Q$ can be rewritten as

$$
Q=Q_{<}+Q_{>}-\sigma
$$

where $Q_{<}$and $Q_{>}$are defined by (A1.3) and (A1.4) respectively with $\alpha=0.1$ is replaced by

$$
\sqrt{\frac{2}{\pi}} \int_{-\infty}^{\infty} \mathrm{d} s \exp \left[-2\left(s-x+\frac{\sigma}{2}\right)^{2}\right]
$$

in (A1.3) and by

$$
\sqrt{\frac{2}{\pi}} \int_{-\infty}^{\infty} \mathrm{d} s \exp \left[-2\left(s-x-\frac{\sigma}{2}\right)^{2}\right]
$$

in (A1.4). This allows to invert the integrations upon $s$ and $x$ in the definitions of $Q_{<}$and $Q_{>}$. By a calculation very similar to the one giving (A1.9), we then obtain

$$
\begin{aligned}
Q=\frac{1}{2} \int_{-\infty}^{\infty} \mathrm{d} s & {\left[\operatorname{Erf}\left(\left(s+\frac{\sigma}{2}\right) \sqrt{2}\right)-\right.} \\
& \left.-\operatorname{Erf}\left(\left(s-\frac{\sigma}{2}\right) \sqrt{2}\right)\right]-\sigma
\end{aligned}
$$

Rewriting the definite integral $\int_{-\infty}^{\infty} \mathrm{d} s .$. involved in (A1.24) as $\lim _{T \rightarrow \infty} \int_{-T}^{T} \mathrm{~d} s \ldots$ and using an integration by parts, we transform (A1.24) as

$$
\begin{array}{r}
Q=-\sqrt{\frac{2}{\pi}} \int_{-\infty}^{\infty} \mathrm{d} s s\left\{\exp \left[-2\left(s+\frac{\sigma}{2}\right)^{2}\right]\right. \\
\left.-\exp \left[-2\left(s-\frac{\sigma}{2}\right)^{2}\right]\right\}-\sigma
\end{array}
$$

The remaining integral $\int_{-\infty}^{\infty} \mathrm{d} s . .$. of $(\mathrm{A} 1.25)$ is obviously equal to $-\sigma \sqrt{\pi} / \sqrt{2}$, and thus $Q$ vanishes.

The derivation of the dipole sum rule (3.7) is very similar to that previously used for the smooth step. The replacement of the constant part (i.e. 1) of $\rho_{\mathrm{B}}(x)$ by the expressions (A1.22) for $x<0$ and (A1.23) for $x>0$ is again the essential trick of the method. Using also

$\lim _{T \rightarrow \infty}[\phi(T)-\phi(-T)]=-4 \int_{-\infty}^{\infty} \mathrm{d} x x q_{\mathrm{B}}(x)$,

we have explicitly checked that

$$
\int_{-\infty}^{\infty} \mathrm{d} x x\left[\rho(x)-\rho_{\mathrm{B}}(x)\right]=0 .
$$

\section{Appendix 2.}

We show that the integral representation (3.4) of $\rho(x)$ satisfies the identity $(4.7)$ when $\rho_{\mathrm{B}}(x)$ is a periodic function with period $\lambda$.

Using (4.6), $\psi(s)$ can be rewritten here as

$$
\psi(s)=\int_{0}^{\lambda} \mathrm{d} u \exp [-\phi(s+u)] G(u),
$$


with

$$
G(u)=\sum_{n=-\infty}^{\infty} \exp \left[-2(u+n \hat{\lambda})^{2}\right]
$$

Using again (4.6), we have

$$
\psi(s+p \hat{\imath})=\psi(s)
$$

for any negative or positive integer $p$. Therefore (3.4) becomes

$$
\rho(x)=\exp [-\phi(x)] \int_{0}^{\lambda} \mathrm{d} s \frac{G(x-s)}{\psi(s)}
$$

Defining

$$
Q_{\lambda}=\int_{0}^{\lambda} \mathrm{d} x\left[\rho(x)-\rho_{\mathbf{B}}(x)\right]
$$

we obtain

$$
Q_{\lambda}=\int_{0}^{\lambda} \mathrm{d} x \rho(x)-\lambda
$$

since the surface charge carried by $-e q_{\mathrm{B}}(x)$ on the interval $[0, \lambda]$ vanishes. Replacing $\rho(x)$ by (A2.4), we rewrite the integral involved in (A2.6) as

$$
\int_{0}^{\lambda} \mathrm{d} s \frac{1}{\psi(s)} \int_{0}^{\lambda} \mathrm{d} x G(x-s) \exp [-\phi(x)]
$$

Making the variable change $v=x-s$ in the integral $\int_{0}^{\lambda} \mathrm{d} x \ldots$, we transform $(\mathrm{A} 2.7)$ as

$$
\begin{gathered}
\int_{0}^{\lambda} \mathrm{d} s \frac{1}{\psi(s)}\left\{\int_{0}^{\lambda} \mathrm{d} v G(v) \exp [-\phi(v+s)]+\right. \\
+\int_{-s}^{0} \mathrm{~d} v G(v) \exp [-\phi(v+s)] \\
\left.+\int_{\lambda}^{\lambda-s} \mathrm{~d} v G(v) \exp [-\phi(v+s)]\right\} .
\end{gathered}
$$

Since $G(u) \exp [-\phi(u)]$ is periodic with period $\lambda$, we see that the two last integrals between the brackets $\{\ldots\}$ in (A2.8) cancel out. The first integral in these brackets is nothing but $\psi(s)$; the total integral (A2.8) reduces then to $\int_{0}^{\lambda} \mathrm{d} s=\lambda$ and we indeed have

$$
\int_{0}^{\lambda} \mathrm{d} x\left[\rho(x)-\rho_{\mathrm{B}}(x)\right]=0 .
$$

\section{References}

[1] Jancovici, B., J. Physique Lett. 42 (1981) L-223.

[2] Jancovici, B., J. Stat. Phys. 28 (1982) 43.

[3] Smith, E. R., J. Phys. A. : Math. Gen. 15 (1982) 1271.

[4] Jancovici, B., J. Stat. Phys. 34 (1984) 803.

[5] Blum, L. and JANCovici, B., J. Phys. Chem. 88 (1984) 2294.

Blum, L., J. Chem. Phys. 80 (1984) 2953.

[6] BLuM, L. and Rosinberg, M. L., to be published.

[7] Ginibre, J. J., J. Math. Phys. 6 (1965) 440.

[8] Menta, M. L., Random Matrices (Academic Press, New York) 1967.

[9] Deutsch, C., De Witt, H. E. and Furutani, Y., Phys. Rev. A 20 (1979) 2631.

[10] Alastuey, A. and Jancovici, B., J. Physique 42 (1981) 1.
[11] Ballone, P., Senatore, G. and Tosi, M. P., Lett. Nuovo Cimento 31 (1981) 619.

[12] Gruber, C., Lebowitz, J. L. and Martin, P. A., J. Chem. Phys. 75 (1981) 944.

Blum, L., Gruber, Ch., Lebowitz, J. L. and Martin, Ph. A., Phys. Rev. Lett. 48 (1982) 1769.

[13] Blum, L., Henderson, D., Lebowitz, J. L., Gruber, C. and Martin, P. A., J. Chem. Phys. 75 (1981) 5974.

[14] Blum, L., Gruber, C., Henderson, D., Lebowitz, J. L. and Martin, P. A., J. Chem. Phys. 78 (1983) 3195.

[15] Jancovici, B., Phys. Rev. Lett. 46 (1981) 386.

[16] Jancovici, B., J. Stat. Phys. 29 (1982) 263.

[17] JANCOVICI, B., private communication.

[18] FORRESTER, P. J., private communication. 